\title{
INSCRIBED AND CIRCUMSCRIBED CIRCLES TO CONVEX CURVES
}

\author{
TUDOR ZAMFIRESCU
}

\begin{abstract}
A convex planar curve may have 2, 3, ., c contact points with its inscribed or circumscribed circle. One of these numbers appears in most cases: 3.
\end{abstract}

Let $\mathcal{C}$ be the space of all closed convex curves in the plane (see [1, p. 3] for a precise definition of a closed convex surface, in particular curve). Several pathological properties of most curves in $\mathcal{C}$ (in the sense of Baire categories) are described in [4] and [5]. We shall show here that most curves in $\mathcal{C}$ have the (expected?) number, 3, of contact points with their inscribed and circumscribed circles. It seems that mainly local properties may be pathological for most curves in e.

We say that most elements of a space of second Baire category have a certain property if those elements which do not have it form a set of first Baire category. Now, $\mathcal{C}$ is of second Baire category (see for instance [5]) if we endow it with the Hausdorff metric, so it makes sense to speak about most curves in $\mathcal{C}$.

Let $C \in \mathcal{C}, D$ be the convex domain with boundary $C, K_{C}$ the circumscribed circle of $C$, i.e. the smallest circle surrounding $D$, and $k_{C}$ an inscribed circle of $C$, i.e. a largest circle included in $D \cup C$. The circle $k_{C}$ is not unique only if $C$ contains parallel segments. Since most curves in $\mathcal{C}$ are strictly convex (see [3] or [2]), they admit a unique inscribed circle.

Clearly, $\operatorname{card}\left(C \cap K_{C}\right)$ may be any cardinal number between 2 and c. We prove

Theorem 1. For most curves $C \in \mathcal{C}, \operatorname{card}\left(C \cap K_{C}\right)=3$.

We will use the following elementary Lemma, that we give without proof.

LeMma. Let $P$ be a polygon such that $P \cap K_{P}$ consists of precisely three points $x_{1}$, $x_{2}, x_{3}$ determining an acute triangle. Let $N_{1}, N_{2}, N_{3}$ be neighborhoods of $x_{1}, x_{2}, x_{3}$. Then there is a neighborhood $\Re$ of $P$ in $\mathcal{C}$ such that, for each $C \in \Re$,

$$
C \cap K_{C} \cap N_{i} \neq \varnothing \quad(i=1,2,3)
$$

and

$$
C \cap K_{C} \subset N_{1} \cup N_{2} \cup N_{3}
$$

Proof of Theorem 1. We first show that the set $C_{2}$ of all curves in $C$ satisfying $\operatorname{card}\left(C \cap K_{C}\right)=2$ is nowhere dense in $\mathcal{C}$.

Received by the editors May 4, 1979.

AMS (MOS) subject classifications (1970). Primary 52A10, 54E52.

(c) 1980 American Mathematical Society 0002-9939/80/0000-0566/\$01.75 
Let $\theta$ be an open set in $\mathcal{C}$. We choose a polygon $P$ in $\theta$.

If $\operatorname{card}\left(P \cap K_{P}\right)=2$, let $x$ and $y$ be the vertices of $P$ lying on $K_{P}$. Let $x_{1}, x_{2}$ be two points such that the segment $x_{1} x_{2}$ contains $x$ and is orthogonal on $x y$. Let $K^{\prime}$ be the circle through $x_{1}, x_{2}, y$. The boundary $P^{\prime}$ of the convex hull of $P \cup\left\{x_{1}, x_{2}\right\}$ has $K^{\prime}$ as circumscribed circle. If $x_{1}$ and $x_{2}$ are close enough to $x, P^{\prime}$ still lies in $\theta$. Obviously

$$
P^{\prime} \cap K^{\prime}=\left\{x_{1}, x_{2}, y\right\} .
$$

If $\operatorname{card}\left(P \cap K_{P}\right) \geqslant 3$, there are three points $x_{1}, x_{2}, x_{3}$ in $P \cap K_{P}$ determining a triangle with all angles of measure at most $\pi / 2$. By gently cutting all the other vertices of $P$ and slightly moving $x_{1}$ if necessary, we obtain a polygon $P^{\prime}$ still belonging to $\theta$ such that $P^{\prime} \cap K_{P^{\prime}}$ is the vertex-set of an acute triangle. Now, by the Lemma, for a neighborhood $\Re$ of $P^{\prime}$ in $\mathcal{C}$, each curve $C \in \Re$ meets $K_{C}$ in at least three points. Thus

$$
\theta \cap \Re \cap C_{2}=\varnothing,
$$

which proves that $C_{2}$ is nowhere dense in $C_{\text {. }}$

Let $e_{(n)}$ be the set of all curves $C$ in $e$ such that

(i) $\operatorname{card}\left(C \cap K_{C}\right) \geqslant 4$, and

(ii) there exist four points $x_{1}, x_{2}, x_{3}, x_{4} \in C \cap K_{C}$ such that the side-lengths of the convex quadrangle with vertices $x_{1}, x_{2}, x_{3}, x_{4}$ are at least $n^{-1}(n \in \mathrm{N})$.

We show that $\mathcal{C}_{(n)}$ is nowhere dense in $\mathcal{C}$.

Let $\theta$ be an open set in $\mathcal{C}$. We choose like before a polygon $P^{\prime}$ in $\theta$ such that $P^{\prime} \cap K_{P^{\prime}}$ is the vertex-set of an acute triangle. Now let $N_{i}$ be a disk of centre $x_{i}$ and radius less than $(2 n)^{-1}$. By the Lemma, there is a neighborhood $\Re$ of $P^{\prime}$ such that, for each curve $C \in \Re$,

$$
C \cap K_{C} \subset N_{1} \cup N_{2} \cup N_{3},
$$

and therefore we cannot find 4 points in $C \cap K_{C}$ determining a convex quadrangle with side-lengths at least $n^{-1}$. Thus

$$
\theta \cap \Re \cap e_{(n)}=\varnothing
$$

and $\mathcal{C}_{(n)}$ is nowhere dense in $\mathcal{C}$.

Let $C_{3}$ be the set of all curves $C \in \mathcal{C}$ verifying $\operatorname{card}\left(C \cap K_{C}\right)=3$. Every curve

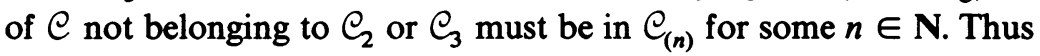

$$
e-e_{3}=e_{2} \cup \bigcup_{n=1}^{\infty} e_{(n)}
$$

where $e_{2}$ and $e_{(n)}(n=1,2,3, \ldots)$ are nowhere dense; therefore $\mathcal{C}-\bigodot_{3}$ is of first Baire category, which proves the theorem.

Surprisingly enough, the proof of Theorem 2 which follows is so similar to the preceding one, that we do not need to give it separately.

Like in the case of $K_{C}, C \cap k_{C}$ may be any cardinal number between 2 and $c$.

Theorem 2. For most curves $C \in \mathcal{C}, \operatorname{card}\left(C \cap k_{C}\right)=3$. 
The above results extend to higher dimensions. Since no technical difficulties appear in connection with increased dimension, we choose to present the planar case as a typical one.

Let $\mathcal{S}^{d}$ be the space of all ( $d$-dimensional) closed convex surfaces $S$ in $\mathbf{R}^{d+1}$. Let $K_{S}$ and $k_{S}$ be the circumscribed and an inscribed hypersphere of $S \in \mathcal{S}^{d}$.

THEOREM 3. For most surfaces $S \in \mathcal{S}^{d}$,

$$
\operatorname{card}\left(S \cap K_{S}\right)=\operatorname{card}\left(S \cap k_{S}\right)=d+2
$$

The proof parallels that of Theorems 1 and 2.

\section{REFERENCES}

1. H. Busemann, Convex surfaces, Interscience, New York, 1958.

2. P. Gruber, Die meisten konvexen Körper sind glatt, aber nicht zu glatt, Math. Ann. 229 (1977), 259-266.

3. V. Klee, Some new results on smoothness and rotundity in normed linear spaces, Math. Ann. 139 (1959), 51-63.

4. R. Schneider, On the curvatures of convex bodies, Math. Ann. 240 (1979), 177-181.

5. T. Zamfirescu, The curvature of mast convex surfaces vanishes almast everywhere (to appear).

Abtrilung Mathematik, Universität Dortmund, 46 Dortmund, Federal Repubuc of Gbruany 